\title{
Blowing up Light: A nonlinear amplification scheme for electromagnetic waves
}

\author{
Ali Mostafazadeh ${ }^{1,2}$, Hamed Ghaemi-Dizicheh ${ }^{2}$, and Sasan Hajizadeh ${ }^{2}$ \\ Departments of Mathematics ${ }^{1}$ and Physics ${ }^{2}$, Koç University, \\ 34450 Sariyer, Istanbul, Turkey
}

\begin{abstract}
We use blow-up solutions of nonlinear Helmholtz equations to introduce a nonlinear resonance effect that is capable of amplifying electromagnetic waves of particular intensity. To achieve this, we propose a scattering setup consisting of a Kerr slab with a negative (defocusing) Kerr constant placed to the left of a linear slab in such a way that a left-incident coherent TE wave with a specific incidence angle and intensity realizes a blow-up solution of the corresponding Helmholtz equation whenever its wavenumber $k$ takes a certain critical value, $k_{\star}$. For $k=k_{\star}$, the solution blows up at the right-hand boundary of the Kerr slab. For $k<k_{\star}$, the setup defines a scattering system with a transmission coefficient that diverges as $\left(k-k_{\star}\right)^{-4}$ for $k \rightarrow k_{\star}$. By tuning the distance between the slabs we can use this setup to amplify coherent waves with a wavelength in an extremely narrow spectral band. For nearby wavelengths the setup serves as a filter. Our analysis makes use of a nonlinear generalization of the transfer matrix of the scattering theory as well as properties of unidirectionally invisible potentials.
\end{abstract}

One of the remarkable properties of nonlinear differential equations is that their initial-value problem may not admit a global solution even if their coefficient functions are smooth. This means that the solution $\psi(x)$ exists in the vicinity of the initial value $x_{0}$ of $x$, but blows up at some $x_{\star}>x_{0}$. These so-called blow-up solutions of nonlinear differential equations have been extensively studied by mathematicians for decades [1-4], but their physical realizations and possible applications have not been fully explored. The purpose of the present article is to outline a concrete physical application of the blow-up solutions which allows for their realization in a scattering setup and forms the basis of a nonlinear amplification scheme for electromagnetic waves.

Consider the time-independent nonlinear Schrödinger equation,

$$
-\psi^{\prime \prime}(x)+\chi(x)\left[\zeta+\gamma|\psi(x)|^{2}\right] \psi(x)=\mathfrak{K}^{2} \psi(x),
$$

where

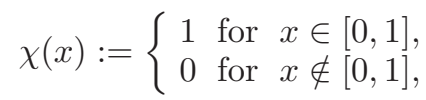

$\zeta, \gamma$, and $\mathfrak{K}$ are real parameters, and $\mathfrak{K}>0$. Suppose that

$$
\gamma>0, \quad \quad \mathfrak{K}^{2}>\zeta,
$$

and let

$$
A:=\sqrt{2\left(\mathfrak{K}^{2}-\zeta\right) / \gamma}, \quad x_{\star}:=\frac{\pi}{2 A \sqrt{2 \gamma}} .
$$

Then it is easy to check that for every phase angle $\varphi$, the function

$$
\psi_{\star}(x):=A e^{i \varphi} \sec \left[\frac{\pi}{4}\left(\frac{x}{x_{\star}}+1\right)\right],
$$

is a solution of (1) in $[0,1]$ provided that $x_{\star}>1$. According to (4), $\psi_{\star}(x)$ blows-up at $x=x_{\star}$. Therefore, it defines a blow-up solution of (1) in $[0,1]$ whenever $x_{\star} \leq 1$.
If $x_{\star}>1$, which means

$$
A<A_{\star}:=\frac{\pi}{2 \sqrt{2 \gamma}}
$$

we can extend (4) to the whole real axis to obtain the following global solution of (11).

$$
\psi_{\star}(x):=\left\{\begin{array}{ccc}
c_{+} e^{i \mathfrak{K} x}+c_{-} e^{-i \mathfrak{K} x} & \text { for } & x<0, \\
A e^{i \varphi} \sec \left[\frac{\pi}{4}\left(x / x_{\star}+1\right)\right] & \text { for } x \in[0,1], \\
d_{+} e^{i \mathfrak{K} x}+d_{-} e^{-i \mathfrak{K} x} & \text { for } & x>1,
\end{array}\right.
$$

where

$$
\begin{aligned}
& c_{ \pm}:=\frac{A e^{i \varphi}}{\sqrt{2}}\left[1 \mp \frac{i \pi(1-\epsilon)}{4 \mathfrak{K}}\right], \\
& d_{ \pm}:=\frac{A e^{i(\varphi \mp \mathfrak{K})} \cos (\pi \epsilon / 4)}{2 \sin ^{2}(\pi \epsilon / 4)}\left[\tan \left(\frac{\pi \epsilon}{4}\right) \mp \frac{\pi i(1-\epsilon)}{4 \mathfrak{K}}\right],
\end{aligned}
$$

$$
\epsilon:=1-A / A_{\star}=1-x_{\star}^{-1} .
$$

so that $\psi_{\star}(x)$ is continuous and differentiable at $x=0$ and $x=1$.

In view of (9)

$$
A=A_{\star}(1-\epsilon), \quad x_{\star}=\frac{1}{1-\epsilon}=1+\epsilon+\mathcal{O}\left(\epsilon^{2}\right),
$$

where $\mathcal{O}\left(\epsilon^{n}\right)$ stands for terms of order $n$ and higher in powers of $\epsilon$. Substituting (10) in the first equation in (3), we have

$$
\mathfrak{K}=\mathfrak{K}_{\star} \sqrt{1-\frac{\pi^{2} \epsilon(2-\epsilon)}{16 \mathfrak{K}_{\star}^{2}}}=\mathfrak{K}_{\star}-\frac{\pi^{2} \epsilon}{16 \mathfrak{K}_{\star}}+\mathcal{O}\left(\epsilon^{2}\right),
$$

where

$$
\mathfrak{K}_{\star}:=\sqrt{\zeta+\frac{\pi^{2}}{16}}
$$




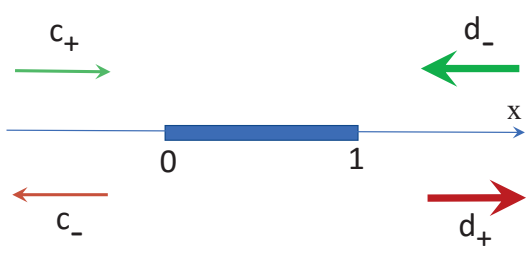

FIG. 1: (Color online) Schematic view of a Kerr nonlinearity confined to a finite interval, i.e., $[0,1]$, on the $x$-axis. $c_{+}$ and $d_{-}$are respectively the complex amplitudes of a pair of left- and right-incident waves. These are scattered into the outgoing waves of amplitude $c_{-}$and $d_{+}$. The thicker arrows represent higher intensity waves.

Expanding the right-hand side of (17) and (8) and making use of (10) and (11), we find

$$
\begin{aligned}
& c_{ \pm}=\frac{A e^{i \varphi}}{\sqrt{2}}\left(1 \mp \frac{i \pi}{4 \mathfrak{K}_{\star}}\right)+\mathcal{O}(\epsilon), \\
& d_{ \pm}=\mp \frac{2 i A e^{i\left(\varphi \mp \mathfrak{K}_{\star}\right)}}{\pi \mathfrak{K}_{\star} \epsilon^{2}}+\mathcal{O}\left(\epsilon^{-1}\right) .
\end{aligned}
$$

According to (9) - (13), if we arrange that $\mathfrak{K} \rightarrow \mathfrak{K}_{\star}$, so that $\epsilon \rightarrow 0, c_{ \pm}$tend to finite values while $d_{ \pm}$diverge quadratically.

The solution (6) corresponds to a situation where a pair of right- and left-going incident plane waves, $c_{+} e^{i(\mathfrak{K} x-\omega t)}$ and $d_{-} e^{-i(\mathfrak{K} x+\omega t)}$, are scattered by a confined nonlinearity [5] into the left- and right-going scattered waves: $c_{-} e^{-i(\mathfrak{K} x+\omega t)}$ and $d_{+} e^{i(\mathfrak{K} x-\omega t)}$, as depicted in Fig. 1. For $\epsilon \approx 0$, the right-incident wave that is sent from $x=+\infty$ and the right-going scattered wave that reaches $x=+\infty$ have a much larger amplitude than the left-incident wave and the left-going scattered wave. This means that the Kerr nonlinearity acts as a filter for the high-intensity incident wave from the right, namely $d_{-} e^{-i(\mathfrak{K} x+\omega t)}$, provided that we inject it from the left by the much lower intensity wave $c_{+} e^{i(\mathfrak{K} x-\omega t)}$. This is actually a curious observation, but is not what we wish to accomplish. Our goal is to explore the possibility of introducing a genuine scattering setup in which a blow-up solution is realized by an incident plane wave that is sent only from the left or the right. The singular nature of the solution would then imply a substantial amplification of the transmitted wave. This signifies a nonlinear amplification scheme that we intend to utilize in optics.

As a first step in this direction, we employ the equivalence of the nonlinear Schrödinger equation (1) with the Helmholtz equation describing the interaction of a transverse electric (TE) wave with a Kerr slab 5 11].

Consider an infinite planar Kerr slab of thickness $L$ that is placed in a nonmagnetic homogeneous linear medium filling the space outside the slab and having a real refractive index $n_{0} \geq 1$. Suppose that we choose a cartesian coordinate system $\{(\mathrm{X}, \mathrm{Y}, \mathrm{Z})\}$ in which the slab occupies the space bounded by the planes $\mathrm{Z}=0$ and $\mathrm{Z}=L$, and $\widehat{\varepsilon}_{l}$ denotes the linear relative permittivity of the slab. Then it is easy to show [10] that the electric field for a time-harmonic TE wave interacting with this system has the form: $\exp \left[i\left(n_{0} k \sin \theta \mathrm{X}-\omega t\right)\right] \mathscr{E}(\mathrm{Z}) \hat{e}_{\mathrm{Y}}$, where $k$ is the wavenumber, $\theta$ is the incidence angle of the wave, $\omega:=c k, c$ is the speed of light in vacuum, $\mathscr{E}(\mathrm{Z})$ is the complex amplitude of the electric field, and $\hat{e}_{j}$ is the unit vector pointing along the $j$-axis for $j=\mathrm{X}, \mathrm{Y}, \mathrm{Z}$. Using Maxwell's equation, we can show that $\mathscr{E}(\mathrm{Z})$ satisfies the Helmholtz equation,

$$
\mathscr{E}^{\prime \prime}(\mathrm{Z})+k^{2} \widehat{\varepsilon}(\mathrm{Z}, \mathscr{E}) \mathscr{E}(\mathrm{Z})=0,
$$

where

$$
\widehat{\varepsilon}(\mathrm{Z}, \mathscr{E}):=\left\{\begin{array}{cc}
\widehat{\varepsilon}_{l}-\sin ^{2} \theta+\sigma|\mathscr{E}(\mathrm{Z})|^{2} & \text { for } \mathrm{Z} \in[0, L], \\
n_{0}^{2}-\sin ^{2} \theta & \text { for } \mathrm{Z} \notin[0, L],
\end{array}\right.
$$

and $\sigma$ is the nonlinearity (Kerr) coefficient. Imposing the electromagnetic interface conditions at the faces of the slab [12], we find that $\mathscr{E}$ and $\mathscr{E}^{\prime}$ must be continuous at $\mathrm{Z}=0$ and $\mathrm{Z}=L$.

In terms of the scaled parameters:

$$
\begin{array}{ll}
x:=\frac{\mathrm{Z}}{L}, & \mathfrak{K}:=k L \sqrt{n_{0}^{2}-\sin ^{2} \theta}, \\
\zeta:=k^{2} L^{2}\left(n_{0}^{2}-\widehat{\varepsilon}_{l}\right), & \gamma:=-k^{2} L^{2} \sigma,
\end{array}
$$

the Helmholtz equation (14) takes the form of the nonlinear Schrödinger equation (11) provided that we set $\psi(x):=\mathscr{E}(L x)$. In view of this relation and Eqs. (53) and (17), (4) gives a blow-up solution of (14), if

$$
\sigma<0, \quad \widehat{\varepsilon}_{l}>\sin ^{2} \theta .
$$

Therefore we need a Kerr slab with negative (defocusing) Kerr coefficient. Refs. [16, 17] study particular examples of Kerr media with negative Kerr coefficient. See alse [18.

According to (3), (15), (10), (16), and (17),

$$
\begin{aligned}
& A=\sqrt{\frac{2\left(\widehat{\varepsilon}_{l}-\sin ^{2} \theta\right)}{-\sigma},} \\
& k=k_{\star}(1-\epsilon), \quad \mathfrak{K}=\tilde{\mathfrak{K}}_{\star}(1-\epsilon),
\end{aligned}
$$

where

$$
k_{\star}:=\frac{\pi}{4 L \sqrt{\widehat{\varepsilon}_{l}-\sin ^{2} \theta}}, \quad \tilde{\mathfrak{K}}_{\star}:=\frac{\pi}{4} \sqrt{\frac{n_{0}^{2}-\sin ^{2} \theta}{\widehat{\varepsilon}_{l}-\sin ^{2} \theta}} .
$$

Substituting (19) in (7) and (8), we find

$$
\begin{aligned}
& c_{ \pm}=\frac{A e^{i \varphi}}{\sqrt{2}}\left(1 \mp \frac{i \pi}{4 \tilde{\mathfrak{K}}_{\star}}\right), \\
& d_{ \pm}=\mp \frac{2 i A e^{i\left(\varphi \mp \tilde{\mathfrak{K}}_{\star}\right)}}{\pi \tilde{\mathfrak{K}}_{\star} \epsilon^{2}}+\mathcal{O}\left(\epsilon^{-1}\right) .
\end{aligned}
$$

Equations (20) and (23) show that $d_{ \pm}$have a quadratic divergence at $k=k_{\star}$. 
Next, we return to the main missing step towards using blow-up solutions for the purpose of amplifying waves, namely devising a genuine scattering system whose transmission coefficient diverges for certain values of the intensity and wavenumber of the incident wave. To do this, first we recall the basic framework for scattering by confined nonlinearities and outline a nonlinear generalization of the transfer matrix of linear scattering theory which proves to be a useful tool for performing the necessary calculations.

Consider the wave equation

$$
-\psi^{\prime \prime}(x)+[v(x)+\mathcal{F}(x, \psi)] \psi(x)=\mathfrak{K}^{2} \psi(x),
$$

where $v(x)$ and $\mathcal{F}(x, \psi)$ are functions representing the linear and nonlinear interactions of a physical system, respectively. Suppose that for $x \rightarrow \pm \infty$ these functions decay to zero at such a rate that the global solutions of (24) tend to plane waves at spatial infinities, i.e.,

$$
\begin{aligned}
& \psi(x) \rightarrow A_{-} e^{i \mathfrak{K} x}+B_{-} e^{-i \mathfrak{K} x} \text { for } \quad x \rightarrow-\infty, \\
& \psi(x) \rightarrow A_{+} e^{i \mathfrak{K} x}+B_{+} e^{-i \mathfrak{K} x} \text { for } \quad x \rightarrow \infty,
\end{aligned}
$$

where $A_{ \pm}$and $B_{ \pm}$are complex coefficients.

The scattering solutions $\psi_{1 / \mathrm{r}}$ of (24) that respectively correspond to a left/right-incident wave of complex amplitude $A^{1 / \mathrm{r}}$ satisfy the asymptotic boundary conditions:

$$
\begin{aligned}
& \psi_{1}(x) \rightarrow\left\{\begin{array}{cl}
A^{1}\left(e^{i \mathfrak{K} x}+R^{\mathrm{l}} e^{-i \mathfrak{K} x}\right) & \text { for } x \rightarrow-\infty, \\
A^{1} T^{\mathrm{l}} e^{i \mathfrak{K} x} & \text { for } x \rightarrow+\infty,
\end{array},\right. \\
& \psi_{\mathrm{r}}(x) \rightarrow\left\{\begin{array}{cl}
A^{\mathrm{r}} T^{\mathrm{r}} e^{-i \mathfrak{K} x} & \text { for } x \rightarrow-\infty, \\
A^{\mathrm{r}}\left(e^{-i \mathfrak{K} x}+R^{\mathrm{r}} e^{i \mathfrak{K} x}\right) & \text { for } x \rightarrow+\infty,
\end{array}\right.
\end{aligned}
$$

where $R^{1 / \mathrm{r}}$ and $T^{\mathrm{l} / \mathrm{r}}$ are respectively the left/right reflection and transmission amplitudes [13, 14]. In the absence of nonlinearity these are complex-valued functions of $\mathfrak{K}$, but in general they depend on both $\mathfrak{K}$ and $A^{1 / \mathrm{r}},[5]$.

The scattering problem defined by (24) admits a transfer-matrix formulation [15]. For a solution specified by its asymptotic form at $x=-\infty$, equivalently the coefficients $A_{-}$and $B_{-}$entering (25), we can identify the transfer matrix with a $2 \times 2$ matrix $\mathbf{M}$ satisfying

$$
\left[\begin{array}{l}
A_{+} \\
B_{+}
\end{array}\right]=\mathbf{M}\left[\begin{array}{l}
A_{-} \\
B_{-}
\end{array}\right] .
$$

For the well-known linear interactions where $\mathcal{F}(x, \psi)=0$, this equation defines $\mathbf{M}$ as a unique $2 \times 2$ matrix that does not depend on $A_{-}$and $B_{-}$. In this case the entries of $\mathbf{M}$ are functions of $\mathfrak{K}$ and its determinant equals unity. In the presence of nonlinearities, det $\mathbf{M}$ may deviate from unity, and the entries of $\mathbf{M}$ depend also on $A_{-}$and $B_{-}$. In this case, (29) does not determine $\mathbf{M}$ in a unique manner, but we can use (27) and (28) to relate any choice of $\mathbf{M}$ satisfying (29) to the reflection and transmission amplitudes in the form

$$
\begin{array}{ll}
R^{\mathrm{l}}=-M_{21}^{\mathrm{l}} / M_{22}^{\mathrm{l}}, & T^{\mathrm{l}}=\operatorname{det} \mathbf{M}^{\mathrm{l}} / M_{22}^{\mathrm{l}}, \\
R^{\mathrm{r}}=M_{12}^{\mathrm{r}} / M_{22}^{\mathrm{r}}, & T^{\mathrm{r}}=1 / M_{22}^{\mathrm{r}},
\end{array}
$$

where $M_{i j}^{1 / \mathrm{r}}$ are the entries of

$$
\mathbf{M}^{l}:=\mathbf{M}\left(A^{l}, A^{l} R^{l}\right), \quad \mathbf{M}^{r}:=\mathbf{M}\left(0, A^{r} T^{r}\right) .
$$

In practice, we can determine $\mathbf{M}\left(A_{-}, B_{-}\right)$for arbitrary choices of $A_{-}$and $B_{-}$by solving the initial-value problem defined by (24) and (25) and using (29). Eqs. (30) and (31) hold for any $\mathbf{M}\left(A_{-}, B_{-}\right)$that we obtain in this way. In view of (32), these provide four complex equations for the four unknowns $R^{l / r}$ and $T^{l / r}$.

An important advantage of the above nonlinear transfer-matrix formulation of scattering theory is that the transfer matrix $\mathbf{M}$ shares the composition property of its linear analog [14, 22 24]. To explain what we mean by this property, suppose that there is a real number $x_{0}$ such that we can decompose the interaction term $v(x)+\mathcal{F}(x, \psi)$ in (24) into the sum of two separate parts, i.e.,

$$
v(x)+\mathcal{F}(x, \psi)=\sum_{j=1}^{2}\left[v_{j}(x)+\mathcal{F}_{j}(x, \psi)\right],
$$

where $v_{1}(x)+\mathcal{F}_{1}(x, \psi)=0$ for $x>x_{0}$ and $v_{2}(x)+$ $\mathcal{F}_{2}(x, \psi)=0$ for $x<x_{0}$. Then we can use (29) to show that the transfer matrix $\mathbf{M}^{(j)}$ associated with the interaction $v_{j}(x)+\mathcal{F}_{j}(x, \psi)$ satisfies

$$
\mathbf{M}^{(2)}\left(A_{0}, B_{0}\right) \mathbf{M}^{(1)}\left(A_{-}, B_{-}\right)=\mathbf{M}\left(A_{-}, B_{-}\right),
$$

where

$$
\left[\begin{array}{l}
A_{0} \\
B_{0}
\end{array}\right]:=\mathbf{M}^{(1)}\left(A_{-}, B_{-}\right)\left[\begin{array}{l}
A_{-} \\
B_{-}
\end{array}\right] .
$$

We refer to (33) as the composition property of nonlinear transfer matrices, and abbreviate it as $\mathbf{M}^{(2)} \circ \mathbf{M}^{(1)}=$ M. For example, consider a case where $\mathcal{F}(x, \psi)=$ $\gamma|\psi(x)|^{2} \chi(x), v(x)=v_{1}(x)+v_{2}(x)$,

$$
\begin{aligned}
& v_{1}(x)=\left\{\begin{array}{cc}
\zeta & \text { for } x \in[0,1], \\
0 & \text { otherwise, }
\end{array}\right. \\
& v_{2}(x)=\left\{\begin{array}{cc}
\mathfrak{z}(x) & \text { for } x \in[a, a+\ell], \\
0 & \text { otherwise, }
\end{array}\right.
\end{aligned}
$$

$\zeta, a$, and $\ell$ are real parameters, $a>1, \ell>0$, and $\mathfrak{z}(x)$ is a real- or complex-valued function. Then we can express the transfer matrix for (24) as

$$
\mathbf{M}=\mathbf{M}^{(2)} \circ \mathbf{M}^{(1)}
$$

where $\mathbf{M}^{(1)}$ and $\mathbf{M}^{(2)}$ are respectively the transfer matrices for the interactions $v_{1}(x)+\gamma|\psi(x)|^{2}$ and $v_{2}(x)$. In particular $\mathbf{M}^{(2)}$ is uniquely determined by the reflection and transmission amplitudes of the finite-range potential $v_{2}(x)$. Denoting these by $R_{2}^{l / r}$ and $T_{2}^{l / r}$, and recalling that scattering potentials enjoy transmission reciprocity [14, 22, 25], so that $T_{2}^{l}=T_{2}^{r}=: T_{2}$, we have

$$
\begin{array}{ll}
M_{11}^{(2)}=T_{2}-R_{2}^{l} R_{2}^{r} / T_{2}, & M_{12}^{(2)}=R_{2}^{r} / T_{2}, \\
M_{21}^{(2)}=-R_{2}^{l} / T_{2}, & M_{22}^{(2)}=1 / T_{2} .
\end{array}
$$




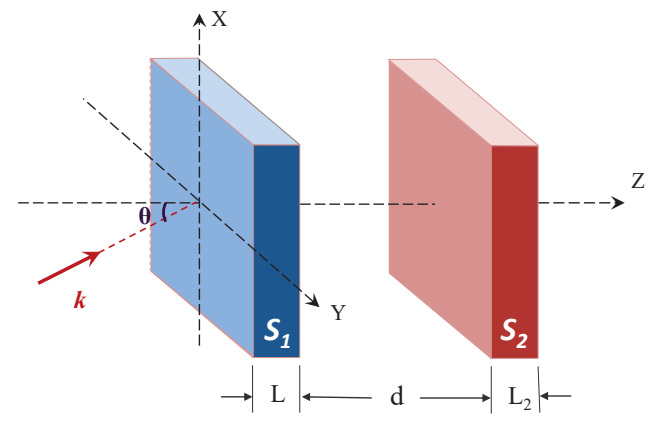

FIG. 2: (Color online) Schematic view of a scattering system consisting of a Kerr slab $\mathcal{S}_{1}$ and a linear slab $\mathcal{S}_{2} . L$ and $L_{2}$ are respectively the thickness of $\mathcal{S}_{1}$ and $\mathcal{S}_{2}$, and $d$ is their distance. $\mathbf{k}$ represents the wavevector for an incoming $\mathrm{TE}$ wave with incidence angle $\theta$.

The scattering setup we outline in the preceding paragraph admits an optical realization involving a homogeneous Kerr slab $\mathcal{S}_{1}$ and a nonmagnetic linear slab $\mathcal{S}_{2}$ that is placed to the right of $\mathcal{S}_{1}$, as depicted in Fig. 2 Again we assume that the space outside the slabs is filled with a homogeneous dielectric medium with a real refractive index $n_{0} \geq 1$, and consider the scattering of the TE waves. Then $a, \ell$, and $\mathfrak{z}(x)$ are related to the distance $d$ between the slabs, and the thickness $L_{2}$ and refractive index $\mathfrak{n}_{2}(x)$ of $\mathcal{S}_{2}$ according to $a=d / L+1, \ell=L_{2} / L$, and

$$
\mathfrak{z}(x)=k^{2} L^{2}\left[n_{0}^{2}-\mathfrak{n}_{2}(x)^{2}\right] .
$$

The Helmholtz equation describing the interaction of the TE waves with this system admits a blow-up solution, if (18) holds. The role of the linear slab is to realize a (near) blow-up solution that fulfills the asymptotic boundary condition (27). In other words, we wish to construct a solution of the form

$$
\psi_{\star}(x):=\left\{\begin{array}{clc}
c_{+} e^{i \mathfrak{K} x}+c_{-} e^{-i \mathfrak{K} x} & \text { for } & x<0, \\
A e^{i \varphi} \sec \left\{\frac{\pi}{4}[(1-\epsilon) x+1]\right\} & \text { for } & x \in[0,1], \\
d_{+} e^{i \mathfrak{K} x}+d_{-} e^{-i \mathfrak{K} x} & \text { for } & x \in(1, a), \\
\phi(x) & \text { for } & x \in[a, a+\ell], \\
c_{+} T^{l} e^{i \mathfrak{K} x} & \text { for } & x>a+\ell,
\end{array}\right.
$$

where $c_{ \pm}$and $d_{ \pm}$are given by (77) and (8), and $\phi(x)$ is the solution of $-\phi^{\prime \prime}(x)+\mathfrak{z}(x) \phi(x)=\mathfrak{K}^{2} \phi(x)$ in $[a, a+\ell]$ that ensures the continuity and differentiability of $\psi(x)$ at $x=a$ and $x=a+\ell$.

According to (29), (36), and (37),

$$
\begin{aligned}
{\left[\begin{array}{c}
c_{+} T^{l} \\
0
\end{array}\right] } & =\mathbf{M}\left[\begin{array}{l}
c_{+} \\
c_{-}
\end{array}\right]=\mathbf{M}^{(2)} \circ \mathbf{M}^{(1)}\left[\begin{array}{l}
c_{+} \\
c_{-}
\end{array}\right]=\mathbf{M}^{(2)}\left[\begin{array}{l}
d_{+} \\
d_{-}
\end{array}\right] \\
& =\frac{1}{T_{2}}\left[\begin{array}{c}
\left(T_{2}^{2}-R_{2}^{l} R_{2}^{r}\right) d_{+}+R_{2}^{r} d_{-} \\
-R_{2}^{l} d_{+}+d_{-}
\end{array}\right] .
\end{aligned}
$$

This in turn implies

$$
R_{2}^{l}=d_{-} / d_{+}, \quad T^{l}=T_{2} d_{+} / c_{+} .
$$

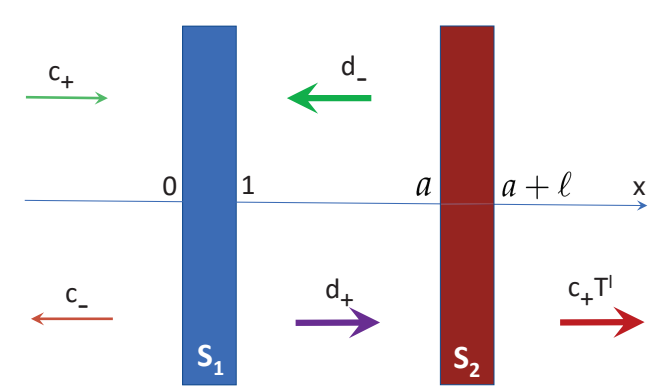

FIG. 3: (Color online) Graphical demonstration of the structure of the solution (39). $S_{1}$ and $S_{2}$ are respectively the nonlinear (Kerr) and linear slabs, which in terms of $x:=\mathrm{Z} / L$, $a=d / L+1$, and $\ell=L_{2} / L$ correspond to intervals $[0,1]$ and $[a, a+\ell]$ on the $x$-axis. $c_{+}, c_{-}$, and $c_{+} T^{l}$ denote the complex amplitude of the incident, reflected, and transmitted waves. Again the thicker arrows represent higher intensity waves.

Substituting (7) and (8) in these equations and making use of (10) and (19), we find that, for $k=k_{\star}(1-\epsilon)$,

$$
\begin{aligned}
R_{2}^{l} & =e^{2 i \tilde{\mathfrak{K}}_{\star}(1-\epsilon)}\left[\frac{4 \tilde{\mathfrak{K}}_{\star} \tan (\pi \epsilon / 4)+i \pi}{4 \tilde{\mathfrak{K}}_{\star} \tan (\pi \epsilon / 4)-i \pi}\right] \\
& =-\exp \left\{2 i \tilde{\mathfrak{K}}_{\star}\left[1-2 \epsilon+\mathcal{O}\left(\epsilon^{2}\right)\right]\right\}, \\
T^{l} & =\frac{T_{2} e^{-i \tilde{\mathfrak{K}}_{\star}(1-\epsilon)}}{\sqrt{2} \sin ^{2}(\pi \epsilon / 4)}\left[\frac{4 \tilde{\mathfrak{K}}_{\star} \sin (\pi \epsilon / 4)-i \pi \cos (\pi \epsilon / 4)}{4 \tilde{\mathfrak{K}}_{\star}-i \pi}\right] \\
& =T_{2}\left[\frac{8 \sqrt{2} e^{-i \tilde{\mathfrak{K}}_{\star}}}{\pi\left(\pi+4 i \tilde{\mathfrak{K}}_{\star}\right) \epsilon^{2}}+\mathcal{O}\left(\epsilon^{-1}\right)\right] .
\end{aligned}
$$

Equation (41) shows that $\left|R_{2}^{l}\right|=1$. If $\mathfrak{z}(x)$ is realvalued, we can use the unitarity condition, $\left|R_{2}^{l / r}\right|^{2}+$ $\left|T_{2}\right|^{2}=1$, to infer that $T_{2}=0$. But it is well-known that the transmission amplitude for a scattering potential never vanishes [14]. This means that in order to realize the near-blow-up scattering solution (39), we must employ a linear medium with a complex refractive index, so that $\mathfrak{z}(x)$ takes complex values and the unitarity relation need not hold. We also demand that for $\mathfrak{K} \approx \mathfrak{K}_{\star}$ (which means $k \approx k_{\star}$ ), the transmission coefficient of this slab is not too small. This implies that $\left|R_{2}^{l}\right|^{2}+\left|T_{2}\right|^{2}>1$. It is not difficult to see that this inequality can be satisfied only if $\mathcal{S}_{2}$ includes gain regions. We give a rigorous proof of this statement in the appendix.

Ref. 27] provides an explicit construction of finiterange potentials with any given reflection and transmission amplitudes at a given wavenumber. Because we need a potential $v_{2}(x)$ that has a sizable transmission amplitude and unit left reflection coefficient, we use a unidirectionally right-invisible potential $u(x)$ with support $[0, \ell]$ and a unit left reflection coefficient at $\mathfrak{K}=\mathfrak{K}_{\star}$. Such a potential fulfills all our requirements except that the phase of its left reflection amplitude may not coincide 
with that of (41). Because translations, $x \rightarrow x-a$, of a finite-range potential change its left reflection amplitude according to, $R^{l} \rightarrow e^{2 i a \mathfrak{K}} R^{l}$, we set

$$
v_{2}(x)=u(x-a)
$$

and adjust $a$ such that the left reflection amplitude of $v_{2}$ at $\mathfrak{K}=\mathfrak{K}_{\star}$ is given by (41). This determines $a$ up to an integer multiple of $\pi / \mathfrak{K}_{\star}$, [27]. We note that adjusting the value of $a$ corresponds to tuning the distance between the slabs. Moreover, because $v_{2}(x)$ is unidirectionally invisible, $T_{2}=1$. Therefore, according to (42), the transmission amplitude of our two-slab system diverges quadratically for $k \rightarrow k_{\star}$. Equivalently, its left transmission coefficient, $\left|T^{l}\right|^{2}$, has a quartic divergence at this wavenumber.

Next, we examine the time-averaged nonlinear relative permitivity of the Kerr slab in the vicinity of its left-hand boundary, i.e., $x=0$. According to (22) and (39), this is given by

$$
\widehat{\varepsilon}_{n l}=\frac{1}{2} \sigma\left|\psi_{\star}(0)\right|^{2}=\sigma A^{2} .
$$

Let us express this quantity in terms of the (timeaveraged) intensity $I$ of the incident wave. To do this we write the refractive index of the Kerr slab at $\mathrm{Z}=0$ in the form $\mathfrak{n}_{1}=\sqrt{\widehat{\varepsilon}_{l}}+n_{2} I$ where $n_{2}$ is a negative real constant. Because typically $\left|n_{2}\right| I \ll \sqrt{\widehat{\varepsilon}_{l}}$ and $\mathfrak{n}_{1}^{2}=\hat{\varepsilon}_{l}+\hat{\varepsilon}_{n l}$, Eq. (44) implies that

$$
|\sigma| A^{2}=\left|n_{2}\right| I\left(2 \sqrt{\widehat{\varepsilon}_{l}}-\left|n_{2}\right| I\right) \approx 2 \sqrt{\widehat{\varepsilon}_{l}}\left|n_{2}\right| I .
$$

Combining this relation with (18) and (19), and noting that $\widehat{\varepsilon}_{n l} \ll 1$, we find: $0<\widehat{\varepsilon}_{l}-\sin ^{2} \theta=|\sigma| A^{2} / 2 \ll 1$. Because $\sin ^{2} \theta<1$, this relation implies that the Kerr slab must be made of a (meta)material whose linear relative permittivity is smaller than unity. Using such a Kerr slab we can realize the proposed nonlinear resonance effect for a TE wave provided that its incidence angle $\theta$ is slightly smaller than $\arcsin \left(\widehat{\varepsilon}_{l}\right)$. In particular for a normally incidence TE wave, we need a metamaterial with a negative Kerr coefficient and a nearly zero linear permittivity [18 21]. Notice, however, that according to (45), $\widehat{\varepsilon}_{l}>n_{2}^{2} I^{2} / 4$.

For a given Kerr slab $\mathcal{S}_{1}$ with $\sigma<0$ and $\widehat{\varepsilon}_{l}<1$, we choose the incidence angle $\theta$ of the TE wave such that $0<\arcsin \left(\widehat{\varepsilon}_{l}\right)-\theta \ll 1$. We can then compute the value of $A, k_{\star}$, and $\tilde{\mathfrak{K}}_{\star}$ using (19) and (21). Next, we choose a linear slab $\mathcal{S}_{2}$ that is unidirectionally right-invisible for $k=k_{\star}(1-\epsilon)$, with $0<\epsilon \ll 1$, and has a unit leftreflection coefficient $\left(\left|R_{2}^{l}\right|^{2}=1\right)$ at this wavenumber. We place $\mathcal{S}_{2}$ at a distance $d$ to the right of $\mathcal{S}_{1}$ such that (41) holds for $k=k_{\star}(1-\epsilon)$. Finally, we prepare a leftincident TE wave with incidence angle $\theta$, wavenumber $k=k_{\star}(1-\epsilon)$, and time-averaged intensity $I$.

It is not difficult to see that the above conditions restrict the thickness $L$ of the Kerr slab. Let $\lambda:=2 \pi / k$ be the wavelength of the incident wave. Then (20), (21), and (44) suggest that

$$
L=\frac{\lambda}{4 A \sqrt{2|\sigma|}}=\frac{\lambda}{4 \sqrt{2\left|\widehat{\varepsilon}_{n l}\right|}} .
$$

Figure 4 shows the plots of the transmission coefficient of our two-slab system for different values of $\epsilon$ and $d$. This corresponds to the scattering of a left-incident wave with intensity $I=1 \mathrm{GW} / \mathrm{cm}^{2}$ where the Kerr slab has thickness $L=503.115 \mu \mathrm{m}$, linear relative permittivity $\widehat{\varepsilon}_{l}=0.25$, and Kerr constant $n_{2}=-10^{-16} \mathrm{~cm}^{2} / W$, so that the incident angle of the wave is to be taken as $\theta=29.999997^{\circ}$, [26]. Both slabs is placed in vacuum, i.e., $n_{0}=1$. The linear slab is modeled using the rightinvisible optical potential (43) with $u(x)$ given by [27]:

$$
u(x):=\left\{\begin{array}{cc}
-\frac{8 \alpha \mathfrak{K}^{2}\left(3-2 e^{2 i \mathfrak{K}_{0} x}\right)}{e^{4 i \mathfrak{K}_{0} x}+\alpha\left(1-e^{2 i \mathfrak{K}_{0} x}\right)^{2}} & \text { for } x \in[0, \ell] \\
0 & \text { otherwise }
\end{array}\right.
$$

where $\alpha=-10^{-4}, \mathfrak{K}_{0}:=\mathfrak{K}_{\star}(1-\epsilon), \ell:=L_{2} / L=$ $400 \pi / \mathfrak{K}_{0}, a=1+d / L, \lambda_{\star}=2 \pi / k_{\star}=900 \mathrm{~nm}$, and

$$
L_{2}=\left\{\begin{array}{l}
189.474 \mu \mathrm{m} \text { for } \epsilon=0.05 \\
200.000 \mu \mathrm{m} \text { for } \epsilon=0.10
\end{array}\right.
$$

As expected $\left|T^{l}\right|^{2}$ has a sharp peak at $\lambda=\lambda_{0}:=$ $2 \pi / k_{\star}(1-\epsilon)$. This is a clear demonstration of the nonlinear resonance effect that we describe above. Notice that for wavelengths slightly different from $\lambda_{0}$ the transmission coefficient takes extremely small values. This shows that our setup acts as a highly effective filter for small deviations from the resonance wavelength $\lambda_{0}$.

The main reason for our choice of (46) for the function $u(x)$ is that it involves the free parameter $\alpha$ which we can tune to set the left reflection coefficient of the linear slab to unity, i.e., make $\left|R^{l}\right|^{2}=1$ for $\lambda=\lambda_{0}$. We can achieve the same purpose using a right-invisible $\mathcal{P} \mathcal{T}$-symmetric bilayer slab whose optical potential is given by (43) and

$$
u(x):=\left\{\begin{array}{cc}
\mathfrak{K}^{2}\left[1-(\eta+i \kappa)^{2}\right] & \text { for } x \in[-\ell / 2,0), \\
\mathfrak{K}^{2}\left[1-(\eta-i \kappa)^{2}\right] & \text { for } x \in[0, \ell / 2], \\
0 & \text { otherwise }
\end{array}\right.
$$

where $\eta$ and $\kappa$ are real numbers determining the refractive index of the two layers as $\eta \pm i \kappa$, and $\ell:=L_{2} / L$. Ref. 28] provides a detailed analysis of the unidirectionally invisible configurations of $\mathcal{P} \mathcal{T}$-symmetric bilayer slabs. This allows for finding right-invisible configuration with unit left reflection amplitude at desired wavelengths $\lambda_{0}$. A simple example is a $\mathcal{P} \mathcal{T}$-symmetric bilayer slab with

$$
\begin{aligned}
& \eta=2.996356, \quad \kappa=3.388790 \times 10^{-3}, \\
& L_{2}=\left\{\begin{array}{l}
318.540 \mu \mathrm{m} \text { for } \epsilon=0.05, \\
301.775 \mu \mathrm{m} \text { for } \epsilon=0.10
\end{array}\right.
\end{aligned}
$$



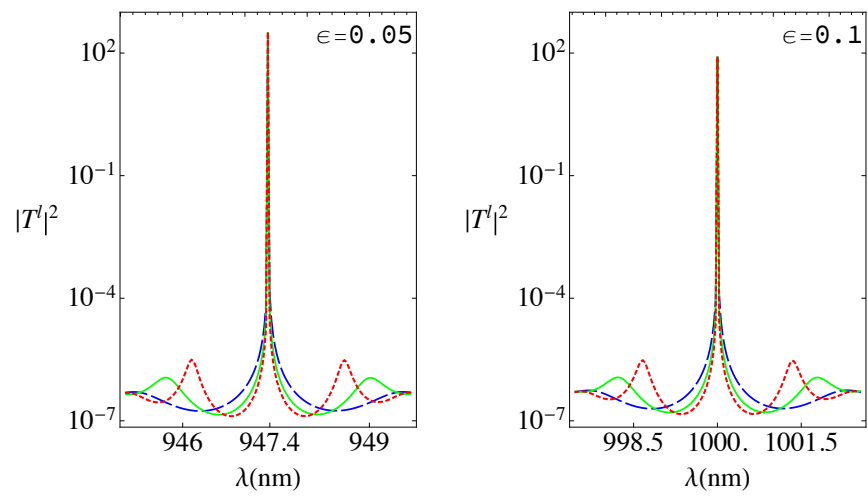

FIG. 4: (Color online) Plots of the transmission coefficient $\left|T^{l}\right|^{2}$ as a function of the wavelength $\lambda$ when the optical potential describing the linear slab is given by (43), (46), and (47). For the graph on the left, $\epsilon=0.05, d=10.162 \mu \mathrm{m}$ (dashed blue curve), $100.162 \mu \mathrm{m}$ (solid green curve), and $200.109 \mu \mathrm{m}$ (dotted red curve). For the graph on the right, $\epsilon=0.10$, $d=10.471 \mu \mathrm{m}$ (dashed blue curve), 100.471 $\mu \mathrm{m}$ (solid green curve), and $200.471 \mu \mathrm{m}$ (dotted red curve). The peak value of $\left|T^{l}\right|^{2}$ are respectively 324.536 and 81.247 for $\epsilon=0.05$ and 0.10. They occur for $\lambda=947.368$ and $1000.000 \mathrm{~nm}$.
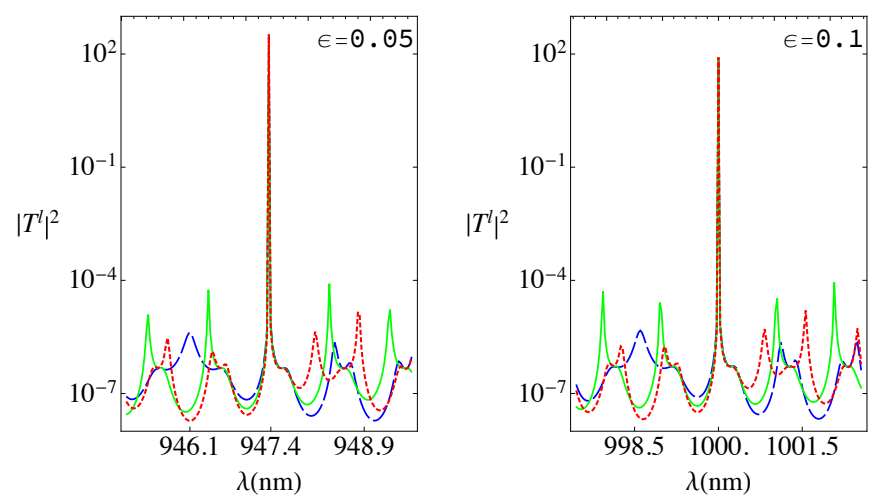

FIG. 5: Plots of the transmission coefficient $\left|T^{l}\right|^{2}$ as a function of the wavelength $\lambda$ when the linear slab is a $\mathcal{P} \mathcal{T}$ symmetric bilayer determined by (43), (48), and (50). For the graph on the left, $\epsilon=0.05, d=10.162 \mu \mathrm{m}$ (dashed blue curve), $100.162 \mu \mathrm{m}$ (solid green curve), and $200.109 \mu \mathrm{m}$ (dotted red curve). For the graph on the right, $\epsilon=0.10$, $d=10.471 \mu \mathrm{m}$ (dashed blue curve), 100.471 $\mu \mathrm{m}$ (solid green curve), and $200.471 \mu \mathrm{m}$ (dotted red curve). The position of the peaks and their height are the same as in Fig. 4. because they are determined by the properties of the incident wave and the Kerr slab.

Figure 5 provides a graphical demonstration of the nonlinear amplification effect for the system depicted in Fig. 2 when we identify the linear slab $\mathcal{S}_{2}$ with the $\mathcal{P} \mathcal{T}$ symmetric bilayer given by (43) and (48) - (50). The physical quantities associated with the incident wave and the Kerr slab are the same as those used to obtain Fig. 4 Comparing Figs. 4 and 5 we see that the choice of the permittivity profile for the linear slab does not affect the general behavior of the system. In particular away from the resonance wavelength $\lambda_{0}$ it displays a strong filtering effect.

A curious question regarding the nonlinear amplification scheme we have developed is weather it complies with the conservation of energy. The scheme amplifies waves which escape to infinity, therefore it should have a source of energy. Because the Kerr slab has a real linear permittivity and Kerr coefficient, it cannot act as an energy source. This suggests that the energy carried away by the amplified wave is to be produced by the linear slab. This indeed agrees with the presence of gain regions in the linear slab. Therefore to maintain its function, we need to pump it with energy. The system spends part of this energy to amplify the transmitted wave. In this sense, the linear slab plays two important roles: 1) It produces the necessary interference effect that eliminates the need for injecting a high-intensity wave form $x=+\infty$ to realize the near blow-up solution; 2) It produces the energy necessary for amplifying the left-incident wave.

The presence of two slabs in our system raises the question whether it is just a laser cavity with $k_{\star}$ being one of its lasing modes. This is actually not true, because the amplification scheme it operates upon is nonlinear, i.e., it only amplifies an incident wave if it has the correct (and sizable) intensity. The basic mathematical concept underlying the amplification effect associated with laser cavities is that of a spectral singularity [22, 29]. This corresponds to the scattering solutions of the linear Helmholtz equation that behave as zero-width resonances. At a spectral singularity both the reflection and transmission amplitudes of the system diverge. Because this happens independently of the amplitude of the incident wave, the system can amplify the background noise to sizable intensities and emit purely outgoing coherent waves. The nonlinear amplification scheme we have outlined in the present article makes use of a fundamentally different mathematical phenomenon, namely the blowup solutions of nonlinear equations. This in particular implies that it cannot be employed to amplify the background noise. It amplifies a left-incident wave only if it has a particular (and generally large) intensity. This in turn implies that one cannot operate the setup for $k=k_{\star}$, because this would give rise to an infinite amplification of an already high-intensity incident wave, which would damage the system. It can only be operated for $k<k_{\star}$ where it would amplify the high-intensity incident wave to a much larger intensity.

An important problem regarding the experimental realizations of our nonlinear amplification scheme is the presence of loses in realistic Kerr slabs, which corresponds to situation where $\widehat{\varepsilon}_{l}$ or $\sigma$ take complex values. This obstructs the exact solvability of the corresponding nonlinear Schrödinger equation [6-8], but does not affect the existence of blow-up solutions as long as the real part of $\sigma$ is negative. Ref. [30] establishes the existence of blow-up solutions for the more general situations where $\widehat{\varepsilon}_{l}$ and $\sigma$ are continuous complex-valued 
functions of $z$ with the real part of $\sigma$ having a negative upper bound, i.e., there is a real number $s_{\max }$ such that $\operatorname{Re}[\sigma(z)] \leq s_{\max }<0$ for all $z \in[0, L]$. In particular, the initial values $\mathscr{E}(0)$ and $\mathscr{E}^{\prime}(0)$ determine a blow-up solution of the Helmholtz equation for such a Kerr slab provided that $\operatorname{Re}\left[\mathscr{E}(0) * \mathscr{E}^{\prime}(0)\right]>0$ and $L \geq 2.023 \times\left\{k^{2}\left|s_{\max }\right| \operatorname{Re}\left[\mathscr{E}(0)^{*} \mathscr{E}^{\prime}(0)\right]\right\}^{-1 / 3}$, [30]. These results provide the theoretical grounds for comprehensive studies of more realistic applications of the nonlinear resonance phenomenon we have introduced in this article.

Acknowledgements: We would like to thank Kaan Güven for suggesting Refs. [19 21], and Varga Kalantarov, Aref Mostafazadeh, and Neslihan Oflaz for illuminating discussions. This work has been supported by the Scientific and Technological Research Council of Turkey (TÜBITAK) in the framework of the project no: 114F357, and by the Turkish Academy of Sciences (TÜBA).

Appendix. A sufficient condition for the presence of gain regions: Consider a possibly complex-valued finiterange potential $v(x)$. Using an appropriate translation and dilation of the independent variable, we can identify the support of this potential with the unit interval, $[0,1]$, i.e., without loss of generality, we suppose that $[0,1]$ is the smallest closed interval outside of which $v(x)$ vanishes. We can use $v(x)$ to describe the interaction of a normally incident TE wave with a planar slab placed in vacuum. Suppose that the slab lies between the planes $\mathrm{Z}=0$ and $\mathrm{Z}=a$ and has a refractive index $\mathfrak{n}(\mathrm{Z})$. Then, $v(x)$ is the optical potential for the slab provided that

$$
\mathfrak{n}(\mathrm{Z})=\sqrt{1-\frac{v(\mathrm{Z} / a)}{\mathfrak{K}^{2}}} .
$$

Here $\mathfrak{K}=k a$ and $k$ is the wavenumber of the incident wave [31]. The Helmholtz equation for this system is equivalent to the Schrödinger equation,

$$
-\psi^{\prime \prime}+v(x) \psi(x)=k^{2} \psi(x) .
$$

Because imaginary part of $\mathfrak{n}(\mathrm{Z})$ is typically much smaller in magnitude than its real part, the regions in which it takes negative values coincide with those where imaginary part of $v(x)$ is positive; $\operatorname{Im}[\mathfrak{n}(\mathrm{Z})]$ and
$\operatorname{Im}[v(\mathrm{Z} / a)]$ have opposite sign. The regions in which $\operatorname{Im}[\mathfrak{n}(Z)]<0$ are called the gain regions, because the propagating waves are amplified while passing through them [31]. We therefore call a region $\mathscr{G}$ of the real axis "a gain region," if $\operatorname{Im}[v(x)]>0$ for all $x \in \mathscr{G}$. Similarly, a lossy region $\mathscr{L}$ is defined by the condition: $\operatorname{Im}[v(x)]<0$ for all $x \in \mathscr{L}$.

Theorem: Let $v(x)$ be a finite-range potential with its left reflection and transmission amplitudes, $R^{l}$ and $T$, satisfying

$$
\left|R^{l}\right|^{2}+|T|^{2}>1
$$

Then the support of $v(x)$ must include gain regions.

Proof: First, we multiply both sides of (52) by $\psi(x)^{*}$ and write the result as

$$
\left[\psi(x)^{*} \psi^{\prime}(x)\right]^{\prime}=\left|\psi^{\prime}(x)\right|^{2}+\left[v(x)-k^{2}\right]|\psi(x)|^{2} .
$$

Evaluating the imaginary part of the left-hand side of this equation and integrating it over the support of $v(x)$, which we identify with $[0,1]$, we find

$$
\left.\operatorname{Im}\left[\psi(x)^{*} \psi(x)^{\prime}\right]\right|_{0} ^{1}=\int_{0}^{1} d x \operatorname{Im}[v(x)]|\psi(x)|^{2} .
$$

Now, consider the case that $\psi(x)$ is a scattering solution of (52) corresponding to a left-incident wave, i.e.,

$$
\psi(x)=\left\{\begin{array}{cc}
A_{-}\left[e^{i k x}+R^{l} e^{-i k x}\right] & \text { for } x \leq 0 \\
A_{-} T e^{i k x} & \text { for } x \geq 1
\end{array}\right.
$$

Substituting this equation in (54) yields

$$
\left|R^{l}\right|^{2}+|T|^{2}-1=\frac{1}{k\left|A_{-}\right|^{2}} \int_{0}^{1} d x \operatorname{Im}[v(x)]|\psi(x)|^{2} .
$$

If the support of $v(x)$ has no gain regions, $\operatorname{Im}[v(x)] \leq 0$ for all $x \in[0,1]$, and the right-hand side of this equation cannot take a positive value. But according to (53), its left-hand side is positive. This implies the presence of gain regions.

It is easy to see that the statement of this theorem also holds for the scattering potentials [14] having an infinite range.
[1] P.-K. Wong, Bounds for solutions to a class of nonlinear second-order differential equations, J. Diff. Eq. 7, 139 (1970).

[2] R. T.Glassey, Blow-up theorems for nonlinear wave equations, Math. Z. 132, 183 (1973).

[3] L. A. Caffarelli and A Friedman, The blow-up boundary for nonlinear wave equations, T. Am. Math. Soc. 297, 223 (1986).

[4] T. Ogawa and Y. Tsutsumi, Blow-up of $H^{1}$ solutions for the nonlinear Schrödinger equation, J. Diff. Eq. 92, 317
(1991).

[5] A. Mostafazadeh, Nonlinear spectral singularities for confined nonlinearities, Phys. Rev. Lett. 110, 260402 (2013).

[6] J. H. Marburger and F. S. Felber, Theory of a lossless nonlinear Fabry-Perot interferometer, Phys. Rev. A 17, 335 (1978).

[7] P. Yeh and M. Khoshnevisan, Nonlinear-optical Bragg scattering in Kerr media, J. Opt. Soc. Am. B 4, 1954 (1987). 
[8] W. Chen and D. L. Mills, Optical response of a nonlinear dielectric film, Phys. Rev. B 35, 524 (1987) and optical behavior of a nonlinear thin film with oblique S-polarized incident wave, 38, 12814 (1988).

[9] P. Peterson, A. Gavrielides, and E. Sakurada, Transverse electric field scattering by a Kerr media deposited on a conducting planar surface, J. Appl. Phys. 68, 446 (1990).

[10] H. Ghaemi-Dizicheh, A. Mostafazadeh, and M. Sarisaman, Nonlinear Spectral Singularities and Laser Output Intensity, J. Opt. 19, 105601 (2017).

[11] A. Mostafazadeh and N. Oflaz, Unidirectional Reflection and Invisibility in Nonlinear Media with an Incoherent Nonlinearity, Phys. Lett. A 381, 3548-3552 (2017).

[12] J. D. Jackson, Classical Electrodynamics (Wiley \& Sons, New York, 1975).

[13] J. G. Muga, J. P. Palao, B. Navarro, and I. L. Egusquiza, Complex absorbing potentials, Phys. Rep. 395, 357-426 (2004).

[14] A. Mostafazadeh, Scattering theory and $\mathcal{P} \mathcal{T}$-symmetry, in Parity-time Symmetry and Its Applications, edited by D. Christodoulides and J. Yang (Springer, to appear), preprint arXiv: 1711.05450 .

[15] A. Mostafazadeh, Nonlinear scattering and its transfer matrix formulation in one dimension, preprint arXiv: 1806.02610.

[16] Y. Li, et al, Polymer-stabilized blue phase liquid crystal with a negative Kerr constant, Opt. Material Express 2, 1135 (2012).

[17] W. Zhang, W. Huang, M. E. Gershenson, and M. T. Bell, Josephson metamaterial with a widely tunable positive or negative Kerr constant, Phys. Rev. Appl. 8, 051001 (2017).

[18] A. D. Neira, N. Olivier, M. E. Nasir, W. Dickson, G. A. Wurtz, and A. V. Zayats, Eliminating material constraints for nonlinearity with plasmonic metamaterials, Nature Comm. 6, 7757 (2015).

[19] R. M. Kaipurath, et al, Optically induced metaltodielectric transition in Epsilon-Near-Zero metamaterials, Sci. Rep. 6, 27700 (2016).

[20] L. Caspani et al, Enhanced nonlinear refractive index in $\epsilon$-Near-Zero materials, Phys. Rev. Lett. 116, 233901 (2016).
[21] M. Z. Alam, I. De Leon, and R. W. Boyd, Large optical nonlinearity of indium tin oxide in its epsilon-near-zero region, Science 352, 795 (2016).

[22] A. Mostafazadeh, Spectral singularities of complex scattering potentials and infinite reflection and transmission coefficients at real energies, Phys. Rev. Lett. 102, 220402 (2009).

[23] L. L. Sánchez-Soto, J. J. Monzóna, A. G. Barriuso, and J. F. Cariñena, The transfer matrix: A geometrical perspective, Phys. Rep. 513191 (2012).

[24] F. Loran and A. Mostafazadeh, Composition of transfer matrices for potentials with overlapping support, Ann. Phys. (NY) 359230 (2015).

[25] Z. Ahmed, Schrödinger transmission through onedimensional complex potentials, Phys. Rev. A 64, 042716 (2001).

[26] These values of $\widehat{\varepsilon}_{l}, n_{2}$, and $I$ together with the identity $|A|=\sqrt{I / \varepsilon_{0} c}$ give $|A|=613.716 \mathrm{kV} / \mathrm{cm}$ and $\sigma=$ $-2.655 \times 10^{-13} \mathrm{~cm}^{2} / \mathrm{kV}^{2}$.

[27] A. Mostafazadeh, Unidirectionally invisible potentials as local building blocks of all scattering potentials, Phys. Rev. A 90, 023833 (2014); Addendum, 90, 055803 (2014).

[28] A. Mostafazadeh, Invisibility and $\mathcal{P} \mathcal{T}$-symmetry, Phys. Rev. A 87, 012103 (2013).

[29] A. Mostafazadeh, Optical spectral singularities as threshold resonances, Phys. Rev. A 83, 045801 (2011);

A. Mostafazadeh and M. Sarisaman, Lasing-threshold condition for oblique TE and TM modes, spectral singularities, and coherent perfect absorption, Phys. Rev. A 91, 043804 (2015).

[30] V. Kalantarov, A. Mostafazadeh, and N. Oflaz, Blowup solutions of Helmholtz equation for a Kerr slab with a complex linear and nonlinear permittivity, preprint arXiv: 1810.03531.

[31] W. T. Silfvast, Laser Fundamentals (Cambridge University Press, Cambridge, 1996).

[32] A. Mostafazadeh, Generalized unitarity and reciprocity relations for $\mathcal{P} \mathcal{T}$-symmetric scattering potentials, J. Phys. A 47, 505303 (2014). 